\title{
Functional microimaging: an integrated approach for advanced bone biomechanics and failure analysis
}

\author{
Romain Voide $^{1}$, G. Harry van Lenthe ${ }^{1}$, Philipp Schneider ${ }^{1}$, Philipp J. Thurner ${ }^{1,2}$, Peter Wyss ${ }^{2}$, Urs \\ Sennhauser $^{2}$, Marco Stampanoni ${ }^{3}$, Martin Stauber ${ }^{1}$, Jess G. Snedeker ${ }^{1}$, \\ Ralph Müller ${ }^{1}$ \\ ${ }^{1}$ Institute for Biomedical Engineering, University and ETH Zürich, Zürich, Switzerland \\ 2 Electronics / Metrology Laboratory, EMPA, Dübendorf, Switzerland \\ ${ }^{3}$ Swiss Light Source, Paul Scherrer Institut, Villigen, Switzerland
}

\begin{abstract}
Biomechanical testing is the gold standard to determine bone competence, and has been used extensively. Direct mechanical testing provides detailed information on overall bone mechanical and material properties, but fails in revealing local properties such as local deformations and strains or quantification of fracture progression. Therefore, we incorporated several imaging methods in our mechanical setups in order to get a better insight into bone deformation and failure characteristics. Our aim was to develop an integrative approach for hierarchical investigation of bone, working at different scales of resolution ranging from the whole bone to its ultrastructure. At a macroscopic level, we used high-resolution and high-speed cameras which drastically increased the amount of information obtained from a biomechanical bone test. The new image data proved especially important when dealing with very small bones such as the murine femur. Here the feedback of the camera in the process of aligning and positioning the samples is indispensable for reproducibility. In addition, global failure behavior and fracture initiation can now be visualized with high temporal resolution. At a microscopic level, bone microstructure, i.e. trabecular architecture and cortical porosity, are known to influence bone strength and failure mechanisms significantly. For this reason, we developed an imageguided failure assessment technique, also referred to as functional microimaging, allowing direct time-lapsed 3D visualization and computation of local displacements and strains for better quantification of fracture initiation and progression at the microscopic level. While the resolution of typical desktop micro-computed tomography is around a few micrometers, highly brilliant X-rays from synchrotron radiation permit to explore the nanometer world. This allowed, for the first time, to uncover fully nondestructively the 3D ultrastructure of bone including vascular and cellular structures and to investigate their role in development of bone microcracks in an unprecedented resolution. We conclude that functional microimaging, i.e. the combination of biomechanical testing with non-destructive 3D imaging and visualization are extremely valuable in studying bone failure mechanisms. Functional investigation of microcrack initiation and propagation will lead to a better understanding of the relative contribution of bone mass and bone quality to bone competence.
\end{abstract}

Key words: Imaging, Biomechanical Testing, Micro-Computed Tomography, Synchrotron Radiation, Bone, Ultrastructure, Fracture, Image-Guided Failure Assessment, Microcrack, Microdamage

\section{INTRODUCTION}

Bone belongs to some of the best investigated biological materials due to its primary function of providing skeletal stability. Bone is susceptible to different local stimuli including mechanical forces and has great capabilities in adapting its mechanical properties to the changes in its environment. Nevertheless, aging or hormonal changes can make bone loose its ability to remodel appropriately, with loss of strength and increased fracture risk as a result. Therefore, bone research is primarily motivated by the social impact and the immense costs in health care associated with osteoporotic fractures [1,2]. Due to the emergence of accurate and precise bone densitometry over the last two decades, bone mass has become a primary endpoint in osteoporosis diagnosis and monitoring. Strong correlations between bone mass and mechanical properties of trabecular bone have been demonstrated for large populations using power-law regressions [39]. However, changes in mass can only partially explain the variation of trabecular bone strength of individuals leaving sometimes up to $90 \%$ of the strength variation unexplained [10]. Thus, accurate diagnosis in a clinical environment based solely on bone densitometry is difficult. With up to $90 \%$ unexplained variation, other factors such as bone

Medical Imaging 2006: Physiology, Function, and Structure from Medical Images, edited by Armando Manduca, Amir A. Amini, Proc. of SPIE Vol. 6143, 61430X, (2006) $\cdot 1605-7422 / 06 / \$ 15 \cdot$ doi: $10.1117 / 12.650485$

Proc. of SPIE Vol. $614361430 X-1$ 
microarchitetcure, bone cell distribution, the distribution of microcracks or microdamage and the quality of the underlying organic bone matrix must play important roles too. These other actors are often referred to as bone quality [11]. However, the relative contribution of bone mass, and bone quality is still poorly understood.

Bone tissue has been studied extensively with micro-computed tomography $(\mu \mathrm{CT})$ and synchrotron radiation based $\mu \mathrm{CT}$ $(\mathrm{SR} \mu \mathrm{CT})$. Tomography using X-rays is a well established technique that is also applied to large systems such as bodyparts or whole organs of human beings [12] and to small systems below micrometer scale such as individual biological cells [13]. Because X-ray based tomography uses the same physical principles at different levels of spatial resolution, $\mu \mathrm{CT}$ and $\mathrm{SR} \mu \mathrm{CT}$ allow investigations of tissues and organs in a hierarchical fashion - from human, to organ, to tissue and down to the individual cell covering more than 6 orders of magnitude in size. Moreover, $\mu \mathrm{CT}$ and SR $\mu \mathrm{CT}$ are non-destructive techniques allowing multiple time-lapsed imaging of samples. Currently the spatial resolution limit for hard X-ray tomography is about $100 \mathrm{~nm}$.

The gold standard to determine bone competence is direct mechanical testing of bone. Direct mechanical testing provides detailed information on overall bone mechanical and material properties, but fails in revealing local functional properties such as local deformations and strains or quantification of fracture progression. In order to get a better insight into bone local deformation and failure characteristics, we combined mechanical testing with imaging to uncover these local properties. Our aim was to develop an integrative approach to hierarchically investigate bone and its mechanical properties, working at different scales of resolution ranging from the whole bone to its ultrastructure. The variety of biomedical imaging techniques makes it possible to work with a wide range of resolution scales, from a macroscopic scale down to the nanometer scale. In the following, a few experiments will be described where both techniques, imaging and biomechanical testing, were combined in a hierarchical fashion. This functional microimaging yielded spectacular insight into bone failure behavior and highlighted the role of bone micro- and ultrastructures in bone strength.

At a macroscopic level, we used an imaging technique to position bone samples in experimental setups and ensure reproducibility of the experiments, since biomechanical tests on small animal bones contain difficulties due to the small size of the samples. High-speed and high-resolution cameras are a very useful and powerful tool in recording biomechanical tests. The informative output of the tests increases drastically when visualizing the whole process from sample preloading to total failure. Bone positioning during preload, global failure and fracture location can be visualized in high temporal resolution.

At the microscopic level, bone samples were imaged in 3D using micro-computed tomography between subsequent micro-compression steps. Bone fracture is a time-dependent, non-linear event including high local deformations and local trabecular fractures. Although some work on bone failure characteristics has been done, basic knowledge of local trabecular failure is still lacking. In estimating the risk of spontaneous fractures, an extended understanding of the failure behavior of trabecular bone is essential. For this reason, our group has developed an image-guided failure assessment (IGFA) technique, also referred to as functional microimaging, allowing investigation of micro-architectural deformations as well as direct time-lapsed 3D visualization and quantification of fracture progression at the microscopic level [14]. This technique has recently been validated as compared to classical continuous mechanical testing $[15,16]$.

In the nano-domain, micro-computed tomography using synchrotron radiation uncovered the ultrastructure of bone and allowed the analysis of microcrack initiation and progression in bone samples under load. Indeed, bone mass and bone architecture are certainly the dominant parameters determining bone strength. However, to determine the strength of the actual bone tissue, cellular effects such as bone mineral density variation or cell lacunae size and distribution must be investigated. Recent results show that bone vasculature is a major contributor to local tissue porosity, and therefore can be directly linked to the mechanical properties of bone tissue [17].

In the current study, all the investigations were performed on murine bones. Indeed, well-characterized mouse lines with phenotypes related to certain aspect of human osteoporosis can be used as an approach to study more homogenous populations in which isolation of candidate chromosomal regions and genetic loci should be faster and more efficient. Animal models complement and extend human studies by allowing close control of environmental factors, by expanding the characterization of phenotypes underlying bone strength and by facilitating breeding strategies to identify genetic linkage.

\section{MATERIAL AND METHODS}

\section{Sample alignment}

Left and right femora from nineteen fresh frozen 16-week-old C57BL/6He (B6) female mice were dissected. They were cut above the proximal condyles, resulting in a length of $11 \pm 0.5 \mathrm{~mm}$. Using a stereomicroscope, the femora were 
positioned in a custom made alignment device, which allowed to simultaneously align 10 samples (Fig. 1a). Online registration of the bones in the alignment device was made with a CCD camera attached to the stereomicroscope and connected to a computer. We performed quantitative assessment of bone alignment by directly evaluating the camera recordings on the computer screen. Quantitative assessment of bone alignment was performed in three measurements. First, we measured the angle $\alpha$ in the transverse plane of the femoral neck loaded in the alignment device, then angles $\beta$ and $\gamma$ of the femoral shaft in the sagittal and frontal planes, respectively (Fig. 1c). Finally, the absolute angle $\delta$ between the vertical axis and the femoral shaft was computed using trigonometry out of $\beta$ and $\gamma$. This new positioning protocol reduced axial alignment $(\delta)$ errors to $1.5^{\circ}$ in the transverse plane, the angle $\alpha$ showed a reproducibility error of $3.23^{\circ}$. After the bones were positioned accurately, they were embedded with cyano-acrylate super-glue (UHU, UHU Schweiz AG, CH-5012 Schönenwerd) into aluminum bone holders which could then be rigidly fixed in the testing device.

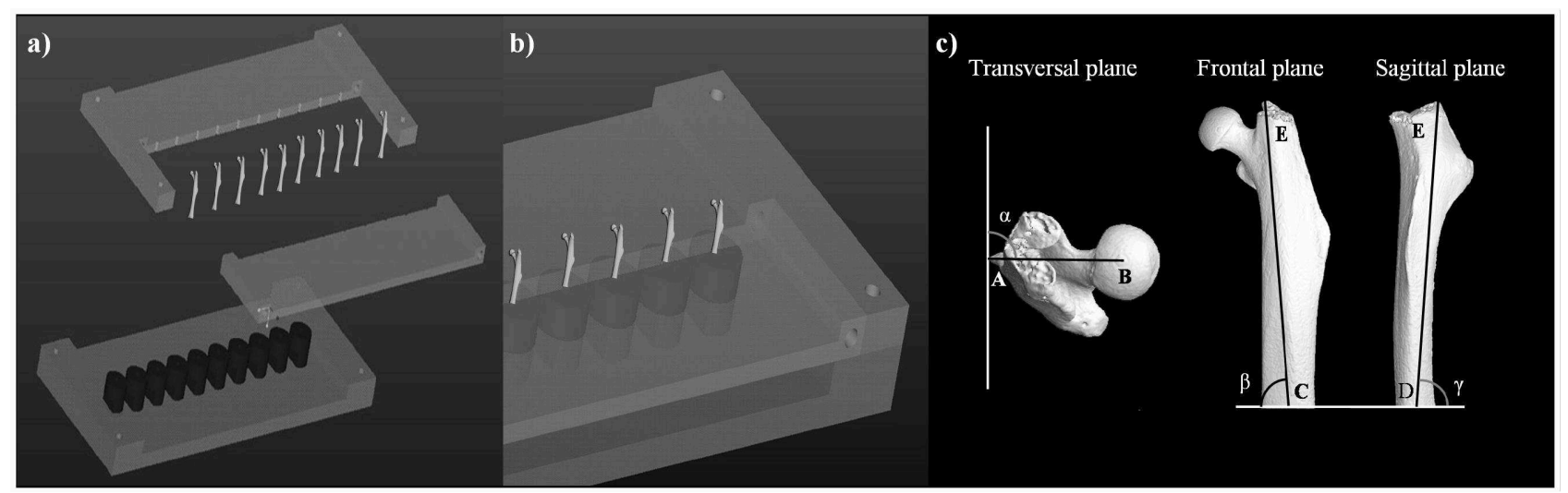

Figure 1: (a) Exploded view of the alignment device with ten murine femora ready to be aligned and ten aluminum cylinders where the femora will be glued into. (b) Detail of the alignment device with aligned bones clamped between the two upper Plexiglas plates. The femora are positioned and glued into aluminum holders that are then rigidly fixed in the materials testing machine. (c) The angular alignment of each murine femur was measured in three planes. left: Angular alignment $\alpha$ in the transverse plane. Natural markers were used to ensure the reproducibility in the alignment protocol from one sample to the other. A: third trochanter, B: center of the femoral head sphere. center: Angular alignment $\beta$ in the frontal plane. C: middle of the femoral shaft at the cut position, E: top of the trochanter. right: Angular alignment $\gamma$ in the sagittal plane. D: middle of the femoral shaft at the cut position, E: top of the trochanter.

\section{Imaging of bone biomechanical testing at the macroscopic level}

At the macroscopic level, we used imaging to obtain additional information from two types of mechanical tests on murine femora: compression at the femoral head and 3-point bending tests. In both loading configurations, tests were imaged with a high-speed and high-resolution camera (AOS Technologies, Daetwil, CH). The image size was set to $1280 \times 1024$ pixels and the frame rate to 62.5 frame/s.

The femoral head compression tests were performed on 16 week old inbred $\mathrm{B} 6$ female mice which were killed by $\mathrm{CO}_{2}$ inhalation. The animals were stored at $-20^{\circ} \mathrm{C}$ and thawed at room temperature just before dissection of the femora. Left and right femora were meticulously prepared in exactly the same way as described in section Sample alignment for mechanical testing. The femora were then rigidly fixed in the materials testing machine (1456, Zwick GmbH \& Co.) (Fig. 2). The mechanical tests consisted of loading the femoral heads until fracture of the femoral neck occurred. The samples were pre-loaded with $1 \mathrm{~N}$ and load-displacement curves were recorded at a crosshead speed of $0.5 \mathrm{~mm} / \mathrm{s}$ [18]. Between 200 and 300 high-resolution images were recorded per single test.

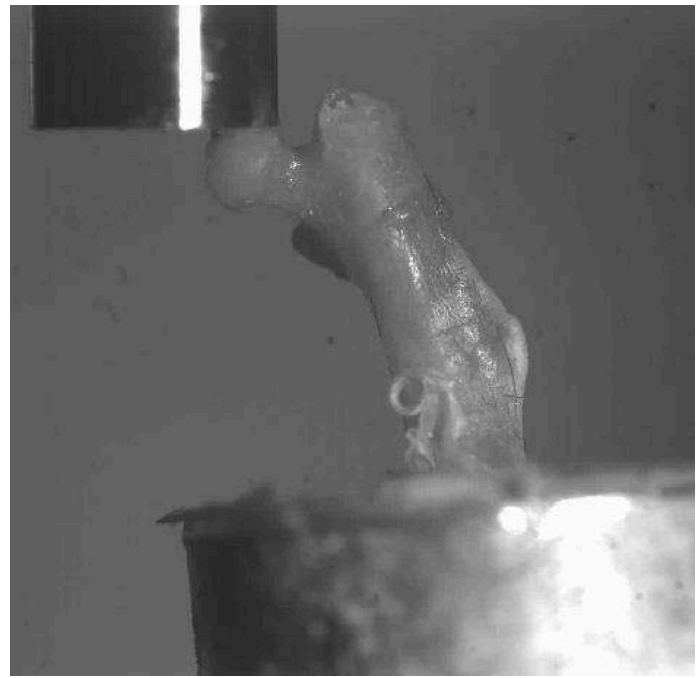

Figure 2: B6 right femur positioned in the materials testing machine under preload of $1 \mathrm{~N}$.

Additionally, femora from 16-week-old B6 as well as from-16-week 
old $\mathrm{C} 3 \mathrm{H} / \mathrm{HeJ}(\mathrm{C} 3 \mathrm{H})$ females and males were tested in three-point bending at room temperature in a custom made loading device. These bones were sized and prepared at the Jackson Laboratory (Main, USA) and kept in alcohol for the oversee travel. Load was applied midway between two supports that were $6 \mathrm{~mm}$ apart. The femora were positioned so that the loading pin was applying a force at a location on the shaft situated at $55 \%$ of the length from the proximal side. The femora were lying freely on the supports and the $1 \mathrm{~N}$ preload oriented them so that the load was applied in the anterior-posterior direction (Fig. 3). Load-displacement curves were recorded at a crosshead speed of $0.5 \mathrm{~mm} / \mathrm{s}$ [18].

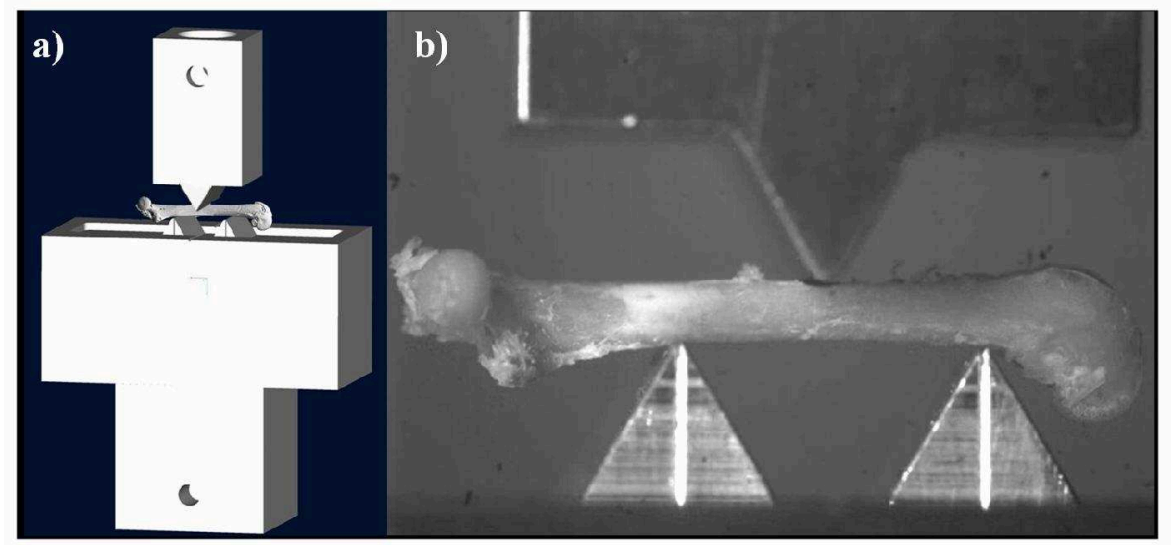

Figure 3: (a) Sketch of a femur loaded in the three-point bending device. (b) Picture of a femur preloaded with 1 N. The supports are $6 \mathrm{~mm}$ apart and the loading pin applies the force at a location on the shaft situated at $55 \%$ of femoral length as measured from the proximal side.

\section{Image-guided failure assessment at the microscopic level}

We performed three types of time lapsed experiments imaged with a desktop $\mu \mathrm{CT}$ ( $\mu \mathrm{CT} 40$, Scanco Medical AG, Bassersdorf, Switzerland). We investigated trabecular aluminum phantoms under compression. The porous alloy was used because its anisotropic and inhomogeneous nature is very similar to trabecular bone found in the human lumbar spine. The samples were cylinders (length: $10 \mathrm{~mm}$, diameter: $7 \mathrm{~mm}$ ) (Fig. 4a) of different densities: 3-5 \%, 7-9 \% and $10-12 \%$. The samples were preloaded with $5 \mathrm{~N}$ and then successively compressed to $1,2,4,8,12$ and $16 \%$ strain. Between each step, the samples were imaged at $20 \mu \mathrm{m}$ resolution.

We also performed femoral head micro-compression tests and three-point bending tests on B6 femora using IGFA. The image resolution for these tests was $20 \mu \mathrm{m}$. The complete compression experiment included 18 steps of $54 \mu \mathrm{m}$ before catastrophic failure of the femoral neck (Fig. 4b). In three-point bending, we loaded the bone in 14 steps up to total failure (Fig. 4c).

In order to reach a higher resolution than with the desktop $\mu \mathrm{CT}$, a loading device was designed to perform IGFA using synchrotron radiation based $\mu \mathrm{CT}$ (SR $\mu \mathrm{CT}$ ) [19] (Fig. 5). We repeated the aforementioned femoral head compressive test with this setup. These tomography experiments were performed at the Material Science (MS) beamline [20] at the Swiss Light Source (SLS) of the Paul Scherrer Institut (PSI). For the IGFA experiments, a photon energy of $20 \mathrm{keV}$ was used. The pixel size in each of the recorded projections was $3.5 \mu \mathrm{m}$. These data were subsequently binned by a factor of two prior to the reconstruction in order to increase the signal to-noise-ratio[21]. At each compression step during IGFA, each sample was scanned 3
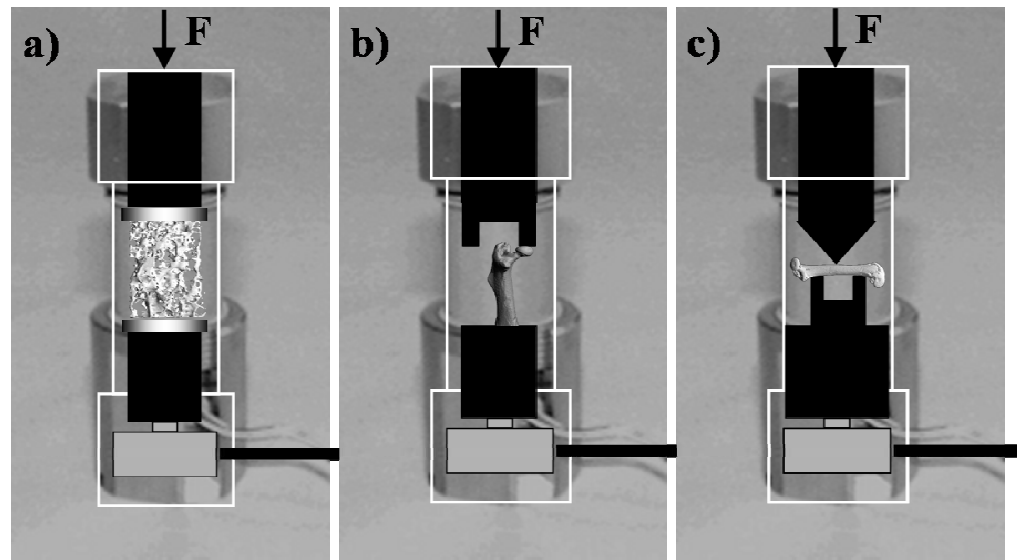

Figure 4: a) Aluminum sample loaded under compression in the IGFA loading chamber. b) B6 femur also loaded under compression and c) B6 femur loaded under three-point bending. times at different heights, since the used $\mathrm{X}$ ray beam was less than $2 \mathrm{~mm}$ in height. The used tomography setup had a field of view (FOV) of $7 \times 7 \mathrm{~mm}^{2}$. Thus, the 
tube made of Torlon 4203 (Solvay S.A., Brussels, Belgium), a poly-amid-imide, around the sample with an outer diameter of $10 \mathrm{~mm}$ was not entirely in the FOV of the detector, hence, local tomography was performed [22].
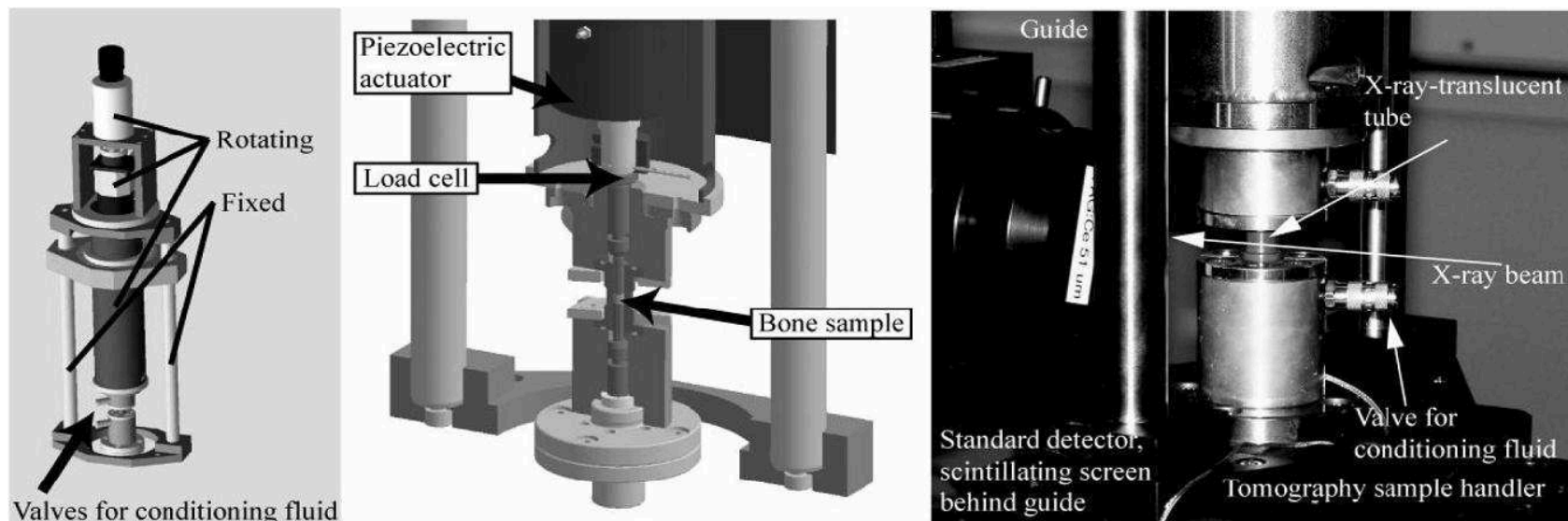

Figure 5: 3D design drawings of the whole loading device (left), and the sample chamber (middle), which is screwed to the actuator housing and the tomography rotation stage. A photograph of the device in operation is given (right) [11].

\section{Image-guided failure assessment in the nano-domain}

Two left femora were taken from two genetically distinct inbred strains of mice $(\mathrm{C} 3 \mathrm{H}$ and $\mathrm{B} 6)$ and were embedded at both ends into polymethyl methacrylate cement (PMMA) (Fig. 6a). We created a round notch in the mid-diaphasis using a high-precision blade saw (Isomet 5000, Bühler LTD, Lake Bluff, Michigan, USA) (Fig. 6b). The notch width was $750 \mu \mathrm{m}$. This notch was expected to create a weakened region in the cortical bone, where the microcracks would initiate and propagate while the bone was loaded. The two samples were then placed into a custom-made loading device, which fitted into the positioning setup of the MS Beamline of the SLS. This loading device comprised an X-ray translucent loading chamber and the sample was loaded under compression with a precision screw. To prevent any torsional constraint due to screwing, a ball bearing was included into the loading device (Fig. 6c). The samples were loaded in a stepwise manner at 1, 2, 4, 8, 12 and $16 \%$ strain. Between each step a high-resolution 3D image (700 $\mathrm{nm}$ nominal resolution) was acquired with the SR $\mu$ CT. A photon energy of $17.5 \mathrm{keV}$ was used. The loading process was deformation controlled. Because of the small FOV of $1.4 \mathrm{~mm} \times 1.4 \mathrm{~mm}$ local tomography was performed.
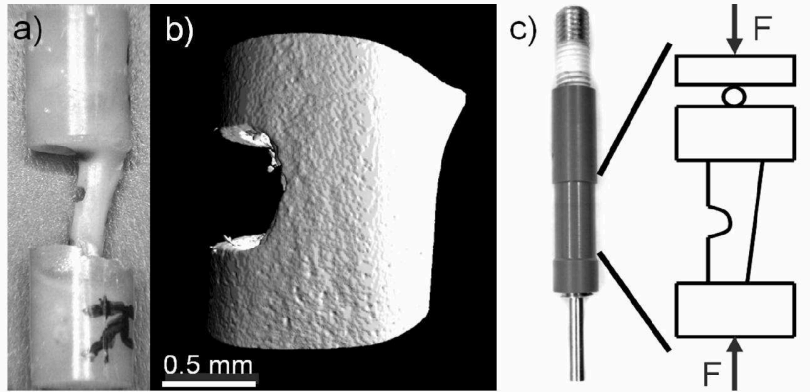

Figure 6: (a) $\mathrm{C} 3 \mathrm{H}$ femur embedded into PMMA, (b) Microtomography 3D image of the notch in the $\mathrm{C} 3 \mathrm{H}$ femur, (c) (left) the loading device with a translucent chamber, a precision screw at the top and (right) the internal setup with a ball joint.

\section{RESULTS}

\section{Imaging of bone biomechanical testing at the macroscopic level}

High-resolution and high-speed imaging during mechanical testing allowed recording of deformations and fractures. We distinguished three characteristic types of fractures. The most frequent one was the oblique fracture going from the proximal side of the femoral head to the distal portion of the neck-shaft jointure $(\mathrm{N}=28)($ Fig. 7a). Then, the second most common fracture type we observed was the so-called trochanteric fracture $(\mathrm{N}=13)$, where the fracture includes a part of the greater trochanter (Fig. 7b). There were also a few vertical fractures which were parallel to the femoral shaft $(\mathrm{N}=8)$ (Fig. 7c). Also in the three-point bending configuration, the additional information gained from the highresolution and high-speed camera revealed the way fractures occurred. The images showed well how bone fractures initiated at the posterior, tensile, side of the bone (Fig. 7d). 

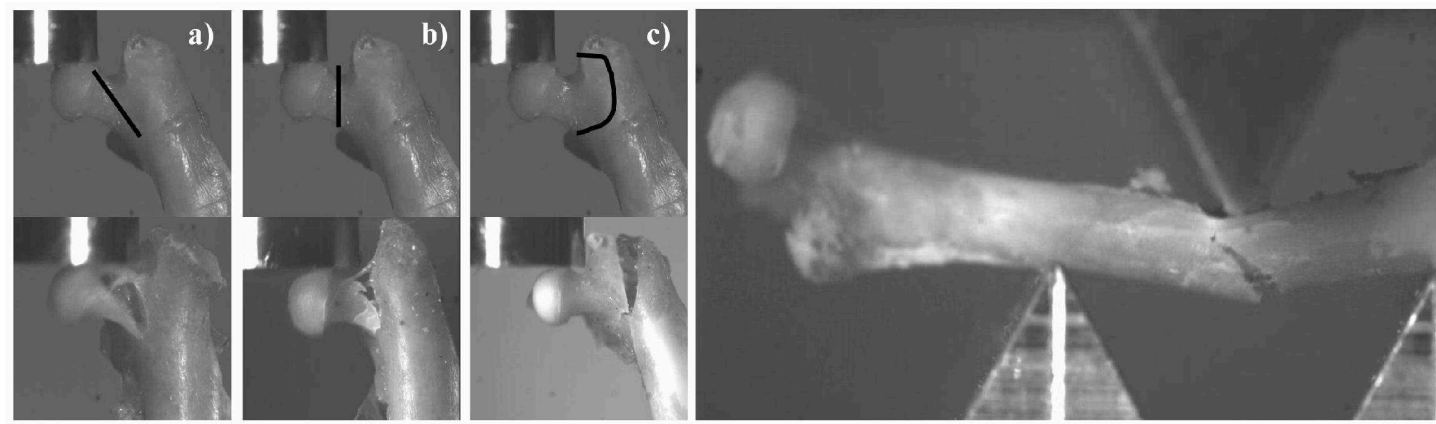

Figure 7: Three characteristic types of femoral neck fractures. (a) oblique, (b) vertical and (c) trochanteric type. (d) In three-point bending, fracture initiates under tension.

Other relevant information delivered by this visualization technique was the exact positioning of the femora in threepoint bending after preload. It provided an easy and elegant way to ascertain that bones, simply put on supports, were positioned correctly and that they rotated in a very reproducible way after preload.

Furthermore, imaging helped to discriminate outliers and permitted to determine if variability in the results was caused by problems in the experimental set-up. Even if a reasonable force versus displacement curve was measured, visualization of the failure process could sometimes identify measurement errors. Indeed, tests where samples slipped in their holder due to bad embedding or with fractures which happened at a wrong location, i.e. femoral head fracture (Fig. 8a), could easily be classified as erroneous tests. Detailed visualization of these tests also permitted to visualize local deformations that were not uncovered by simple analysis of the test outputs such as femoral head deformation under compression (Fig. 8b) or local cortical deformations at the supports in three-point bending (Fig. 8c).
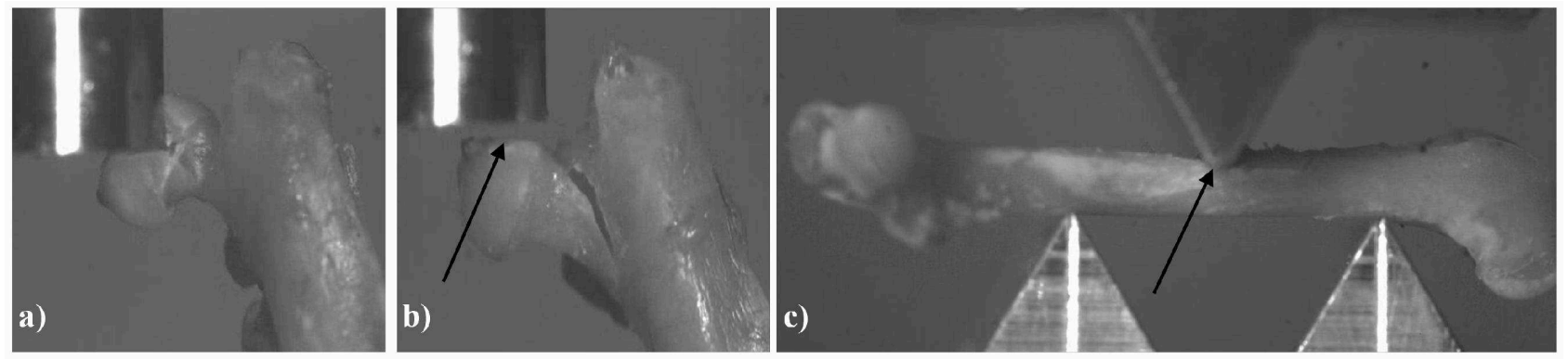

Figure 8: (a) Bad fracture occurring in the femoral head instead of femoral neck. (b) Local deformation of the femoral head at the loading pin contact surface. The femoral head sphere is clearly flattened (black arrow). (c) Local deformation of cortical bone in the femur midshaft in a three-point bending test (black arrow).

\section{Image-guided failure assessment at the microscopic level}

Performing IGFA on trabecular aluminum phantoms showed local deformations as well as overall deformation and failure mechanisms of the loaded sample. The 3D visualization allowed observing local processes like single trabecula deforming under tension or compression or ongoing buckling. Most of the tested samples showed a global deformation plane at a $45^{\circ}$ angle. The recorded force versus deformation curves allowed identifying the transition from elastic to plastic deformation (Fig. 9b). 

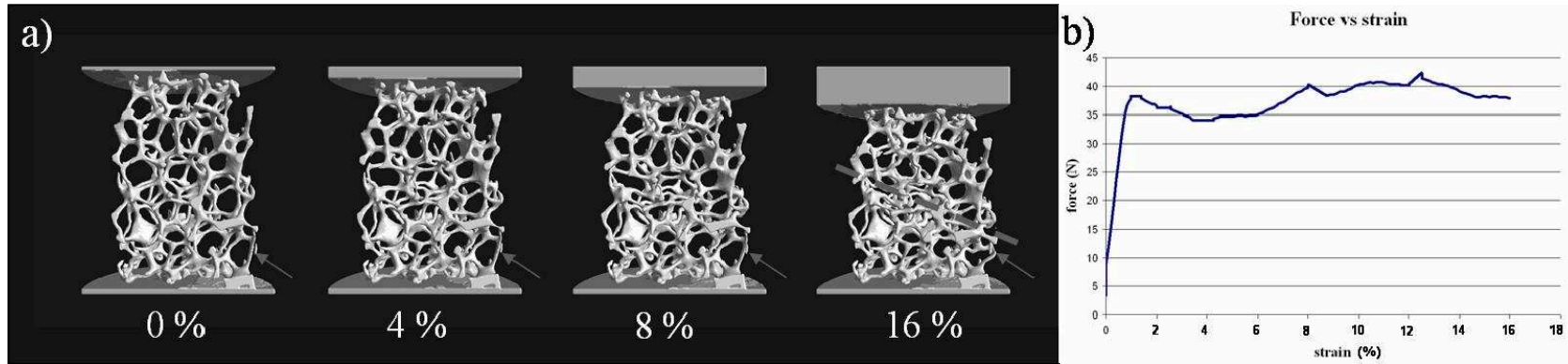

Figure 9: (a) Middle density sample. Compression steps from 0 to $16 \%$ with characteristic deformation pattern $45^{\circ}$ tilted plane (dashed line) and typical trabecula buckling (arrow). (b) Force - strain curve of this sample.

The failure visualization technique allowed a good insight into the structural failure mechanism of whole inbred murine femora (Fig. 10a). We could follow the deformation of the trabeculae and the formation of cracks in the cortical bone as well as in the trabecular bone. The mechanical properties of the bone were derived from the force-displacement diagram (Fig. 10b).
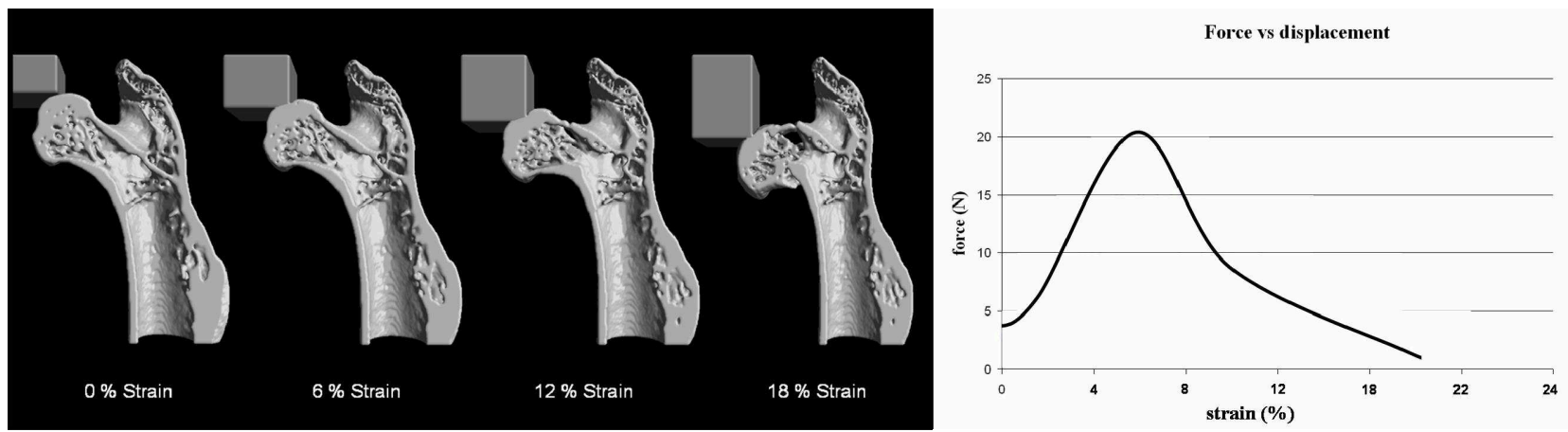

Figure 10: (left) Assessment of femur deformation pattern under axial loading. Initial, intermediate and final steps of IGFA testing on a mouse femur. (right) Typical force-displacement diagram for compression testing at the femoral head.

In the same fashion as with the high-resolution, high-speed camera, IGFA showed relevant details of the tests that could not be deduced from the quantitative output only. In addition to uncover the structure failure mechanism, IGFA also imaged the local deformations at the interface between bone samples and loading setups such as femoral head deformation under compression (Fig. 11a) or local cortical deformations at the supports in three-point bending (Fig. 11b).

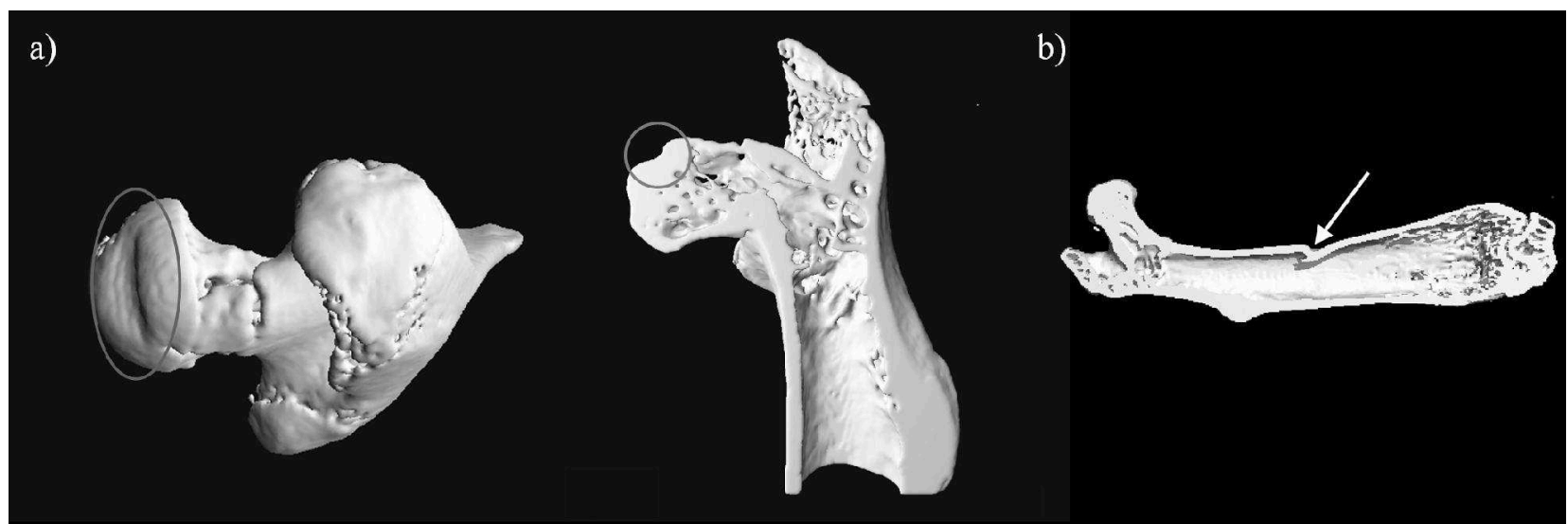

Figure 11: (a) Local deformation of the femoral head at the loading pin contact. Formation of a notch due to the loading pin (grey circles). (b) Local deformation of cortical bone in the femur midshaft in a three-point bending test (white arrow). 
In order to visualize trabecular structure in more detail, as well as to better identify crack initiation and crack growth, IGFA was also performed using $\mathrm{SR} \mu \mathrm{CT}$. Femoral head compression tests were imaged at $3.5 \mu \mathrm{m}$ resolution. Despite a longer imaging time and a more complicated setup than common IGFA, images assessed by SR $\mu \mathrm{CT}$ showed more details with better quality (Fig. 12). Fracture initiation and propagation are

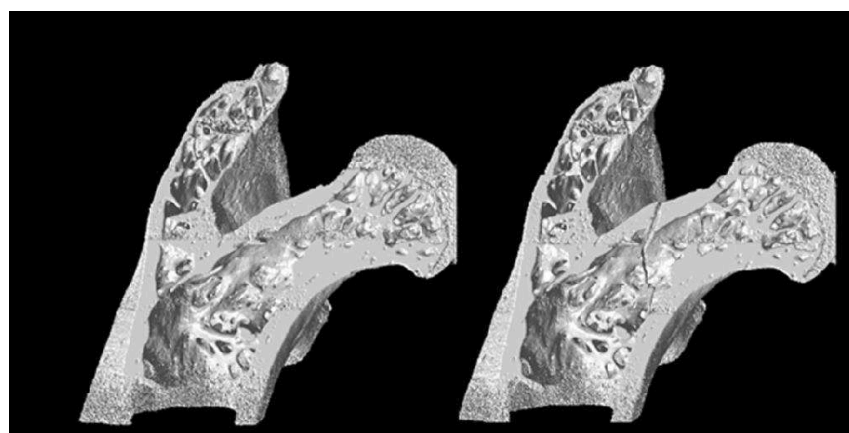

$0 \mu \mathrm{m}$

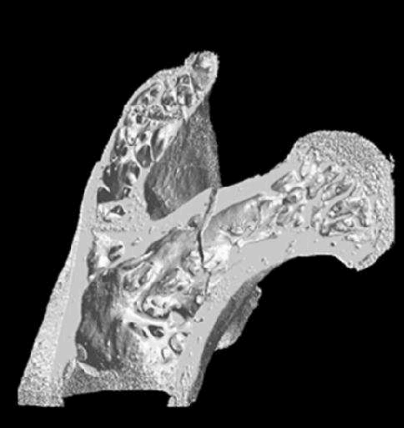

$60 \mu \mathrm{m}$ very well depicted in such experiments.

\section{Image-guided failure assessment in the nano-domain}

Initiation and propagation of microcracks were imaged at a nanoscopic resolution in a non-destructive manner. This experiment showed microcracks initiation while increasing the sample strain. Cortical bone of the B6 sample showed no microcrack up to $2 \%$ apparent strain. At $4 \%$ apparent strain, microcracks appeared at several locations (Fig. 13a, b).

In the $\mathrm{C} 3 \mathrm{H}$, we investigated the microcracks behavior in more detail (Fig. 13 c-f). The analysis revealed that microcracks propagation is usually not stopped by the lacunae; microcracks went through these osteocytic cavities (Fig. 13f). Moreover SR $\mu \mathrm{CT}$ images at $700 \mathrm{~nm}$ resolution permitted a superb visualization of uncracked ligament bridging, which are thought to be of extreme importance in the bone toughening mechanism (Fig. 13e).
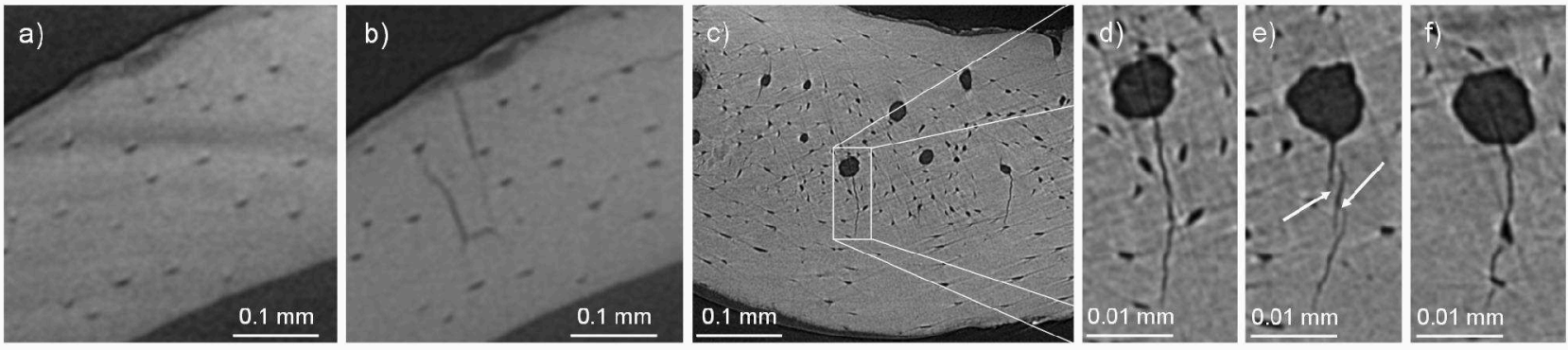

Figure 13: SR $\mu \mathrm{CT}$ reconstructed slices of B6 cortical bone close to the notch. Nominal resolution: $700 \mathrm{~nm}$, binned. (a): $2 \%$ apparent strain, no microcrack, (b): 4\% apparent strain, microcrack initiation. (c-f) SR $\mu \mathrm{CT}$ reconstructed slices of $\mathrm{C} 3 \mathrm{H}$ cortical bone. Nominal resolution: $700 \mathrm{~nm}$. (c) Region close to the notch; the small spots are osteocyte lacunae and the bigger ones are vessels, (d) Blow-up of identified region in fig 13c: crack initiation in the vessel, (e) Uncracked ligament bridging along the crack path (arrows), (f) The microcrack propagates through two lacunae.

3D SR $\mu \mathrm{CT}$ imaging also allowed to visualize the microcracks in different planes, e.g. in the microcrack wake plane or perpendicular to it. So, it is now possible to observe microcracks in all orientations (Fig. 14).

Figure 14: (a) Segmented 3D image of microcracks wakes in different planes. (b) 3D cut at the level of the black line in (a), (c) Same as (b) with semitransparent bone.

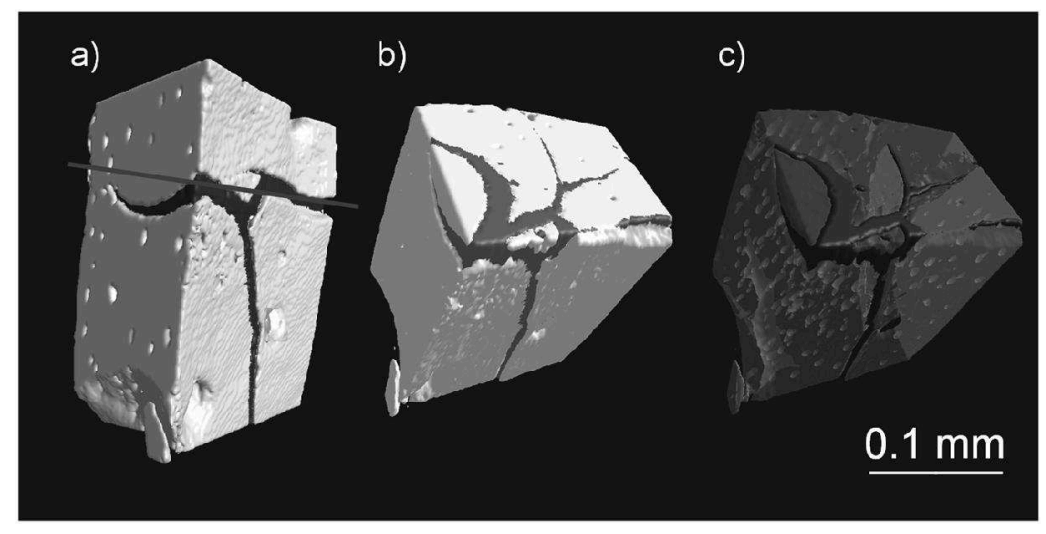




\section{DISCUSSION}

In this paper, we described an integrative approach for hierarchical bone investigation, working at different scales of resolution ranging from the whole bone to its ultrastructure. We combined several imaging methods with standard mechanical setups in order to get a better insight into bone deformation and failure mechanisms.

The imaging setup to perform better bone alignment for mechanical testing helped to gain a very accurate positioning ensuring excellent reproducibility. The axial alignment error of only $1.5^{\circ}$ in angle $\delta$ is very encouraging. This is of outmost importance since several studies have shown that the stiffness of proximal femur is very dependent on the loading direction [23-25]. Indeed, in accordance with these studies, our own data (data not shown) demonstrated that a few degrees change in the angle of inclination of the femoral midshaft led to significant changes in the femoral neck mechanical properties. The alignment device had an angular reproducibility error of $3.23^{\circ}$ in the transversal plane. Although being larger than the axial alignment error, this error is of lesser importance for common vertical compressive tests of the femoral neck, since, in this configuration, the femur is vertically oriented and the mechanical setup is axis symmetric with the respect to vertical axis.

Macroscopic imaging of common mechanical tests, such as proximal femur compression or three-point bending, drastically increased the informative output of these tests. We could improve the experimental reproducibility by removing bad samples (Fig. 8a) and easily recognize outliers. As a consequence, variability of the tests was reduced. This is of great value in experimental studies, because reduced variability means increased power in the results. This means that fewer samples and therefore fewer animals are needed to obtain significant results.

Looking at local deformations at loading or support points, visualization with high-speed, high-resolution cameras permitted to correct the measured total deformation of the sample. Indeed, classical tests do not take in account that local deformations form at contact points between load and samples (Fig. 8b, d). These local deformations are then automatically added and the measured total deformation is not the intended deformation.

Macroscopically investigating typical failure locations and fracture propagation gave a first insight into bone failure behavior. In combination with finite element modeling, which can be used to accurately determine stress and strain fields, these types of observations provided precious information to relate fracture initiation to stress and strain; hence, will be of great value in assessing yield parameters for bone.

Moving to microscopic scales and investigating bone at a microstructural level allowed further observations on why and how bone fails. As discussed before, bone mass alone does not explain entirely variations in bone strength and competence. Preliminary data have shown that predicting bone strength can be greatly improved by including microarchitectural parameters in the analysis $[16,26,27]$. IGFA is therefore a very powerful tool in visualizing and quantifying local behavior of bone microstructure under load. This technique showed trabecular deformation under compression (Fig. 9) as well as whole bone behavior with its internal microstructure changes during femoral head compression or three-point bending tests (Fig. 10-12). Adding to this tool a novel image analysis approach to compute local displacements and strains in the compressed structures [28], close tracking of very local structural changes, such as single trabecular deformation is now possible.

In the same manner as already mentioned for macroscopic visualization of bone deformations, IGFA is also very helpful when working with finite element models of bone under load. Analyzing local deformations and strains is of tremendous importance in assessing yield parameters for bone. These parameters are then included in $\mu$ FE-models [29]. Further, IGFA samples are excellent samples to develop and validate $\mu \mathrm{FE}$-models. With $\mu \mathrm{FE}$ meshes generated directly from the same 3D microstructural images as the ones produced during IGFA, we can simulate mechanical tests in great details and with high precision. Comparing these models with the results obtained in IGFA, i.e. visually and computationally comparing the deformations obtained with $\mu \mathrm{FE}$ and IGFA, allows validating or invalidating new types of bone microstructural finite element models, such as non-linear $\mu \mathrm{FE}$ models or beam-like models $[16,30]$.

Exploration of bone in the nano-domain is a necessity when investigating bone competence. Bone is an inhomogeneous material with a complex hierarchical microstructure [31-34] that can be considered at several dimension scales. With $\mathrm{SR} \mu \mathrm{CT}$, we are now able to visualize the so-called bone ultrastructure. Ultrastructure, comprising vasculature, osteocytic lacunae and micro-damage, is thought to play an important role in bone material and mechanical properties as well as in bone remodeling processes. In order to fully understand bone competence, bone as a material has to be characterized first. And there, the ultrastructure certainly plays a key role. Bone porosity and the distribution of its different entities (vessels, lacunae, canaliculi and micro damage) are factors that directly influence material properties [35]. Furthermore, bone ultrastructure is meant to be partly responsible for bone remodeling and has active contributions to mechanisms such as mechanotransduction; osteocytes are believed to be distributed inside bone in a way to best sense the local environment and load applied on bone [35-38]. 
Focusing on the micro-damage effects, we concluded from our nanoscopic investigation of bone, that microcracks were often initiated in vessel spaces (Fig. 13d-f) and were not stopped by osteocytic lacunae, but propagated easily through these smaller voids (Fig. 13f), demonstrating the strong influence of bone ultrastructure, especially vasculature, on crack development and propagation. Moreover SR $\mu$ CT images at $700 \mathrm{~nm}$ resolution permitted a superb visualization of uncracked ligament bridging, which is thought to be of extreme importance in the bone toughening mechanism (Fig. 13e). With the obtained submicron resolution, we are now able to observe very small features such as uncracked ligament bridging as well as the microcrack wake path through lacunae in a $3 \mathrm{D}$ manner. This method provides a great basis for future 3D microdamage quantification.

Many studies have investigated the role of microdamage in bone resistance. Microcrack initiation and the related uncracked ligament bridging are thought to be responsible for bone toughening mechanisms [19, 34, 39-43]. This toughening mechanism could play an important role in determining bone's resistance to fracture during fatigue. It is well known that cyclic loading in bone results in degradation of mechanical properties including strength and stiffness, leading ultimately to fatigue failures, at load levels well below the fracture loads [44-47]. Indeed, "stress" fractures of human bone are a well recognized clinical problem with incidence rates of 1-4\% often being reported [48-50]; even higher rates are cited for adolescent athletes and military recruits [51, 52]. Such fractures are believed to result from continued repetitive loading and the induced microdamage accumulation. Accumulaiton of microdamage may lead to loss of bone quality and increase the risk of osteoporotic fractures [53, 54].

In this study, we showed that $\mathrm{SR} \mu \mathrm{CT}$ imaging in the nano-domain is a very powerful tool to investigate initiation, propagation and damage accumulation of microcracks (Fig. 13). The non-destructive characteristics of this technique permits to effectively visualize and quantify propagation and evolution of microcracks under dynamic loading. Moreover, the 3D approach uncovers the crack wake under different orientations and this is an important advantage in comparison with other 2D imaging methods (Fig. 14). Microcraks and microdamage seem then to be deeply involved in different mechanisms that directly affect bone competence. Therefore, our tool to investigate bone in $3 \mathrm{D}$ and in an unprecedented nanoscopic volumetric resolution contains a huge potential in trying to answer all those questions and validate or invalidate the many hypothesis found in literature concerning bone ultrastructure and bone quality.

In summary, we have developed in this study an integrative method to investigate bone competence in a hierarchical fashion. We were able to observe at the whole bone level how overall fractures initiate and propagate. On the microscopic scale, we examined how the bone internal microstructure behaves when bone is under load. Local deformations and microarchitectural changes were observed and analyzed. Nano-tomography permitted to look into the ultrastructure of bone. Further work in this high resolution field will provide very important clues on bone intrinsic material properties and on ultrastructure contribution to overall bone failure behavior.

\section{CONCLUSION}

This paper presents for the first time an integrative image-guided approach to measure and analyze bone failure initiation and propagation in a fully non-destructive way, and even more importantly, in a hierarchical fashion ranging from macro to nano; from organ to cell.

The developed integrative approach to functionally and hierarchically determine bone competence is very powerful. Enormous potential to investigate bone properties and bone competence is still hidden in these new techniques. The time-lapsed high resolution 3D imaging of bone under load will provide better understanding of bone failure processes by non-invasive assessment of local deformation, fracture initiation and fracture growth, which cannot be assessed with other techniques in an integrative fashion.

\section{ACKNOWLEDGMENTS}

This study was funded by ETH Intramural Funding (TH 00124/41-2631.5) and Swiss National Science Foundation through the SNF Professorship in Bioengineering (FP 620-58097.99, PP-104317/1).

\section{REFERENCES}

1. $\quad$ Ray, N.F., et al., Medical expenditures for the treatment of osteoporotic fractures in the United States in 1995: report from the National Osteoporosis Foundation. J Bone Miner Res, 1997. 12(1): p. 24-35. 
2. Cooper, C., et al., Population-Based Study of Survival after Osteoporotic Fractures. Am J Epidemiol, 1993. 137(9): p. 1001-5.

3. McElhaney, J.H., et al., Mechanical properties of cranial bone. J Biomech, 1970. 3(5): p. 495-511.

4. Carter, D.R. and W.C. Hayes, The compressive behavior of bone as a two-phase porous structure. Journal of Bone and Joint Surgery [Am], 1977. 59(7): p. 954-962.

5. Williams, J.L. and J.L. Lewis, Properties and an anisotropic model of cancellous bone from the proximal tibial epiphysis. J Biomech Eng, 1982. 104(1): p. 50-56.

6. Rice, J.C., S.C. Cowin, and J.A. Bowman, On the dependence of the elasticity and strength of cancellous bone on apparent density. Journal of Biomechanics, 1988. 21(2): p. 155-168.

7. Odgaard, A. and F. Linde, The underestimation of Young's modulus in compressive testing of cancellous bone specimens. Journal of Biomechanics, 1991. 24(8): p. 691-698.

8. $\quad$ Keller, T.S., Predicting the compressive mechanical behavior of bone. J Biomech, 1994. 27(9): p. 1159-68.

9. $\quad$ Keaveny, T.M., et al., Trabecular bone exhibits fully linear elastic behavior and yields at low strains. Journal of Biomechanics, 1994. 27(9): p. 1127-36.

10. Ciarelli, M.J., et al., Evaluation of orthogonal mechanical properties and density of human trabecular bone from the major metaphyseal regions with materials testing and computed tomography. J Orthop Res, 1991. 9(5): p. 674-82.

11. Thurner, P.J., et al., Novel Techniques for High-Resolution Functional Imaging of Trabecular Bone. Proceedings of SPIE, Medical Imaging 2005: Physiology, Function and Structure from Medical Images, 2005. 5746: p. 515-526.

12. Marincek, B., P.R. Ros, and M. Reiser, Multislice CT - A Pratical Guide. 2000, Berlin: Springer.

13. Weiss, D., et al., Computed tomography of cryogenic biological specimens based on X-ray microscopic images. Ultramicroscopy, 2000. 84: p. 185-97.

14. Müller, R., S.C. Gerber, and W.C. Hayes, Micro-compression: a novel technique for the nondestructive assessment of local bone failure. Technol Health Care, 1998. 6(5-6): p. 433-44.

15. Nazarian, A. and R. Müller, Time-lapsed microstructural imaging of bone failure behavior. J Biomech, 2004. 37(1): p. 55-65.

16. Müller, R., et al. Functional microimaging at the interface of bone mechanics and biology. in 10th Mediterranean Conference of the International Federation for Medical and Biological Engineering (IFMBE). 2004. Ischia, Italy.

17. Schneider, P., et al., Soft Tissue and Phase Contrast Imaging at the Swiss Light Source. Proceedings of SPIE, Medical Imaging 2004: Physiology, Function and Structure from Medical Images, 2004. 5368: p. 281-291.

18. Turner, C.H., et al., Genetic regulation of cortical and trabecular bone strength and microstructure in inbred strains of mice. J Bone Miner Res, 2000. 15(6): p. 1126-31.

19. Thurner, P., et al., An integrated loading and staining device for micrometer resolution image-guided failure assessment of bone using synchrotron light. Bone (conditionnaly accepted), 2005.

20. Stampanoni, M., et al., High resolution X-ray detector for synchrotron-based microtomography. Nuclear Instruments \& Methods in Physics Research Section a-Accelerators Spectrometers Detectors and Associated Equipment, 2002. 491(1-2): p. 291-301.

21. Thurner, P., B. Muller, and F. Beckmann. An optimization procedure for spatial and density resolution in hard X-ray micro-computed tomography. 2004.

22. Weitkamp, T., et al. In-line phase contrast in synchrotron-radiation microradiography and tomography. in Developments in X-ray Tomography III. 2002. San Diego: SPIE.

23. Carpenter, R.D., et al., New QCT analysis approach shows the importance of fall orientation on femoral neck strength. J Bone Miner Res, 2005. 20(9): p. 1533-42.

24. Pinilla, T.P., et al., Impact direction from a fall influences the failure load of the proximal femur as much as age-related bone loss. Calcif Tissue Int, 1996. 58(4): p. 231-5.

25. Bergmann, G., et al., Hip contact forces and gait patterns from routine activities. J Biomech, 2001. 34(7): p. 859-71.

26. Goldstein, S.A., The mechanical properties of trabecular bone: dependence on anatomic location and function. J Biomech, 1987. 20(11-12): p. 1055-61.

27. Turner, C.H., et al., The fabric dependence of the orthotropic elastic constants of cancellous bone. J Biomech, 1990. 23(6): p. 549-61. 
28. Müller, R., et al., Micro-mechanical evaluation of bone microstructures under load., in Developments in X-Ray Tomography III, U. Bonse, Editor. 2002, SPIE: San Diego, CA. p. 189-200.

29. Pistoia, W., et al., Estimation of distal radius failure load with micro-finite element analysis models based on three-dimensional peripheral quantitative computed tomography images. Bone, 2002. 30(6): p. 842-8.

30. Stauber, M., et al., A finite element beam-model for efficient simulation of large-scale porous structures. Comput Methods Biomech Biomed Engin, 2004. 7(1): p. 9-16.

31. Currey, J., 'Osteons' in biomechanical literature. J Biomech, 1982. 15(9): p. 717.

32. Rho, J.Y., L. Kuhn-Spearing, and P. Zioupos, Mechanical properties and the hierarchical structure of bone. Med Eng Phys, 1998. 20(2): p. 92-102.

33. Weiner, S., W. Traub, and H.D. Wagner, Lamellar bone: structure-function relations. J Struct Biol, 1999. 126(3): p. 241-55.

34. Nalla, R.K., et al., Mechanistic aspects of fracture and R-curve behavior in human cortical bone. Biomaterials, 2005. 26(2): p. 217-31.

35. Vashishth, D., et al., Decline in osteocyte lacunar density in human cortical bone is associated with accumulation of microcracks with age. Bone, 2000. 26(4): p. 375-80.

36. Belanger, L.F., C. Belanger, and T. Semba, Technical approaches leading to the concept of osteocytic osteolysis. Clin Orthop Relat Res, 1967. 54: p. 187-96.

37. Shea, J.F., V.L. Yeager, and J.J. Taylor, Bone resorption by osteocytes. Proc Soc Exp Biol Med, 1968. 129(1): p. 41-3.

38. Burr, D.B., A.G. Robling, and C.H. Turner, Effects of biomechanical stress on bones in animals. Bone, 2002. 30(5): p. 781-6.

39. Burr, D.B., et al., Bone microdamage and skeletal fragility in osteoporotic and stress fractures. J Bone Miner Res, 1997. 12(1): p. 6-15.

40. Sobelman, O.S., et al., Do microcracks decrease or increase fatigue resistance in cortical bone? J Biomech, 2004. 37(9): p. 1295-303.

41. Nalla, R.K., J.J. Kruzic, and R.O. Ritchie, On the origin of the toughness of mineralized tissue: microcracking or crack bridging? Bone, 2004. 34(5): p. 790-8.

42. Diab, T. and D. Vashishth, Effects of damage morphology on cortical bone fragility. Bone, 2005. 37(1): p. 96102.

43. Nalla, R.K., et al., Effect of aging on the toughness of human cortical bone: evaluation by R-curves. Bone, 2004. 35(6): p. 1240-6.

44. Blickenstaff, L.D. and J.M. Morris, Fatigue fracture of the femoral neck. J Bone Joint Surg Am, 1966. 48(6): p. 1031-47.

45. Carter, D.R. and W.C. Hayes, Compact bone fatigue damage--I. Residual strength and stiffness. J Biomech, 1977. 10(5-6): p. 325-37.

46. Schaffler, M.B., E.L. Radin, and D.B. Burr, Mechanical and morphological effects of strain rate on fatigue of compact bone. Bone, 1989. 10(3): p. 207-14.

47. George, W.T. and D. Vashishth, Damage mechanisms and failure modes of cortical bone under components of physiological loading. J Orthop Res, 2005. 23(5): p. 1047-53.

48. Nalla, R.K., et al., Aspects of in vitro fatigue in human cortical bone: time and cycle dependent crack growth. Biomaterials, 2005. 26(14): p. 2183-95.

49. Burr, D.B., Bone, exercise, and stress fractures. Exerc Sport Sci Rev, 1997. 25: p. 171-94.

50. Taylor, D., Failure processes in hard and soft tissues, in Comprehensive structural integrity: Fractures of materials from nano to macro, I. Milne, R. Ritchie, and B. Karihaloo, Editors. 2003, Elsevier Inc.: Oxford, UK. p. 35-96.

51. Meurman, K.O. and S. Elfving, Stress fracture in soldiers: a multifocal bone disorder. A comparative radiological and scintigraphic study. Radiology, 1980. 134(2): p. 483-7.

52. Iwamoto, J. and T. Takeda, Stress fractures in athletes: review of 196 cases. J Orthop Sci, 2003. 8(3): p. $273-8$.

53. Burr, D.B., et al., Does microdamage accumulation affect the mechanical properties of bone? J Biomech, 1998. 31(4): p. 337-45.

54. Vashishth, D., et al., In vivo diffuse damage in human vertebral trabecular bone. Bone, 2000. $26(2)$ : p. 147-52. 